\title{
PERSONALEINSATZPLANUNG FÜR BODENVERKEHRSDIENSTE - Ein exakter Algorithmus
}

\author{
Ralf Szymanski
}

\section{Zusammenfassung}

Die Bodenverkehrsdienste (BVD) bewältigen auf den Flughäfen die Frachtgut- bzw. Gepäckabfertigung, die technischen Abfertigungen und die Innenreinigung von Flugzeugen. Neben den qualitativen Standards ist es zunehmend wichtiger, betriebswirtschaftliche effiziente Strukturen aufzubauen.

Ziel der Personaleinsatzplanung ist es, die nichtproduktiven Wartezeiten zwischen den Abflügen zu minimieren. Ein kostenminimaler Einsatzplan bestimmt den Arbeitsbeginn sowie die Arbeitsdauer des Personals - also wann, wie viele Personen für wie lange zu arbeiten haben.

Es werden Modelle präsentiert, die auf variablen Arbeitsschichten des Personals von mindestens 3-Stunden - bis maximal 10-Stunden-Schichten basieren. Ein Schwerpunkt ist die Analyse unterschiedlicher Mindestdauern der Arbeitszeiten von 3, 31/2 und 4 Stunden. Die Lösungen der Modelle werden mit einem exakten mathematischen Verfahren auf einem handelsüblichen PC berechnet.

Bei einem realen Rechenbeispiel der Be- und Entladung von Flugzeugen wurden die Modelle mit unterschiedlichen Mindestschichtdauern verglichen. Es zeigte sich ein Unterschied von $7,8 \%$ bei den Personalkosten und $3,4 \%$ bei der Arbeitsproduktivität, wobei sich das 3-Stunden-Modell als effizienter erwies.

\begin{abstract}
Ground Air Scheduling handles work tasks like cabin cleaning, loading, etc. at terminal airports. A high quality standard and efficient structures become more and more important.

Personal scheduling performs the minimization of the nonproductive time between the flights. So the resulting schedule minimizes costs. It specifies how many employees are needed - including start and end working time.

Specific models contain a variable working time from 3 to 10 hours a day. Different minimum working time of $3,3 \frac{1}{2}$ und 4 hours are analyzed. The solution processes are done with a discrete mathematical algorithm on a standard PC.

A real life example of loading an aircraft with different minimum working times is processed. The minimum working time of 3 hours saves $7.8 \%$ costs respectively $3.4 \%$ productivity.
\end{abstract}

\section{»I. EINLEITUNG}

Der Betrieb von Flughäfen beinhaltet eine Vielzahl von Planungsaufgaben. Eine davon ist die Planung des Personaleinsatzes der Bodenverkehrsdienste (BVD). Die Tätigkeiten der BVD umfassen das Betanken, Rangieren, Reinigen sowie die Be- und Entladung von Flugzeugen. Aufgrund dieser Aufgaben ist es nachvollziehbar, das ca. 2/3 der Mitarbeiter eines Flughafens im Bereich der BVD tätig sind (Newsroom ADV deutsche Verkehrsflughäfen 2012b).

Die aktuelle Situation ist durch eine geplante Neuregelung der EU-Kommission geprägt. Bei der ersten Marktöffnung durch die EU-Kommission 1996 gelang es, die Preise der BVD um bis zu $25 \%$ zu senken. Hauptursächlich ist dies durch die Möglichkeit eines zweiten BVD-Dienstes für die Flughäfen ermöglicht worden. Bezüglich der Personaleinsatzplanung bedeutet jeder weitere BVD-Anbieter an einem Flughafen eine potentielle Verschlechterung. Denn viele Abfertigungen sind relativ leicht zu planen, weil das notwendige Personal kontinuierlich beschäftigt wird. Seit der Marktöffnung von 1996 müssen die BVD-Anbieter nun mehr fragmentierte Arbeitseinsätze berücksichtigen - also Arbeitseinsätze, mit nicht durchgängig zu beschäftigten Mitarbeitern. Somit ist ein entscheidender Indikator die Arbeitsproduktivität bzw. die effektive Arbeitszeit, die ein Mitarbeiter aktiv beschäftigt werden kann. Aufgrund des personalintensiven Aufgabenbereichs bestreiten die ca. 20.000 BVD-Mitarbeiter in Deutschland ca. 70\% der Gesamtkosten der BVDAnbieter (Newsroom ADV deutsche Verkehrsflughäfen 2012a/b).
Es ist zu befürchten, dass weitere Kosteneinsparungen zu einer qualitativen Verschlechterung der Abfertigungsprozesse führen. Auslösende Faktoren hierfür sind der internationale Wettbewerb im Luftverkehr sowie das Kostenmanagement der Fluggesellschaften.

\section{» II. PERSONALEINSATZPLANUNG FÜR BODENVERKEHRSDIENSTE}

Die Herausforderung eines optimalen Personaleinsatzes für die BVD ist es, die nichtproduktive, wartende Arbeitszeit zu minimieren. Dies ist ideal, wenn es sehrviele oder sehrwenige abzufertigende Flugzeuge gibt. Schwieriger wird die Situation, wenn es unvermeidliche Wartezeiten geben muss. Eine Möglichkeit wäre es, dies durch zeitspezifische Abfertigungsgebühren direkt an den Kunden 
weiterzureichen bzw. die Planung der Personaleinsätze zu verbessern. Rahmenbedingungen sind tarifliche und gesetzliche Bestimmungen zu der Anzahl von Mindeststunden je Schicht sowie den Pausenregelungen eines Mitarbeiters. Im Folgenden werden die Be- und Entladung von abzufertigenden Flugzeugen betrachtet. Da es sich hierbei um eine computergestützte Planung handelt, gilt es - wie generell bei Softwareprojekten - der Spezifikation von Datenschnittstellen die größtmögliche Aufmerksamkeit und Sorgfalt zu widmen. Selbstverständlich sollten die Datenschnittstellen so beschaffen sein, dass zukünftige Änderungen bzw. Ergänzungen ohne großen Aufwand erfolgen können. Außerdem sollen die Strukturen denen des bestehenden Arbeitsablaufs ähneln, da somit Eingabefehler reduziert werden.

Die Mitarbeiter werden aktuell in drei Qualifikationskategorien eingeteilt, wobei jede eine beispielhafte spezifische Entlohnung in Euro [€] je Stunde [h] beinhaltet, die auch Zuschläge für Feiertags-, Wochenend- und Nachtschichten ermöglicht:

\begin{tabular}{|c|c|c|c|c|c|}
\hline Id & Bezeichnung & $€ / \mathrm{h}$ & Feiertag & Wochenende & Nacht \\
\hline LM & Loadmanager & $15,-$ & $+50 \%$ & $+50 \%$ & $+25 \%$ \\
\hline FA & Flugabfertiger & $10,-$ & $+50 \%$ & $+50 \%$ & $+25 \%$ \\
\hline LO & Loader & $8,-$ & $+50 \%$ & $+50 \%$ & $+25 \%$ \\
\hline
\end{tabular}

Zusätzlich sind beispielhafte Pausenregelungen in Abhängigkeit der Arbeitszeit zu erfassen:

\begin{tabular}{|c|c|}
\hline Arbeitszeit in [h] & Pausenlänge \\
\hline$\leq 4,5$ & 0 Minuten \\
\hline$\leq 6$ & 15 Minuten \\
\hline$\leq 9$ & 30 Minuten \\
\hline$>9$ & 60 Minuten \\
\hline
\end{tabular}

Ein weiterer Parameter begrenzt für die Optimierung die maximale Nettoarbeitszeit auf 10,5 Stunden.

Aus Gründen der Benutzer- bzw. Bedienfreundlichkeit des Systems gibt es ein dreistufiges Konzept zur Eingabe der Arbeitseinsätze für die Mitarbeiter (je Qualifikationsstufe).

Als Standard gibt es die Hinterlegung der Arbeitsminuten vor und nach einer Ankunft sowie vor und nach eines Abflugs pro Flugzeugtyp. Zusätzlich gibt es die Option, fluglinienspezifische Informationen je Flugzeugtyp einer Airline zu hinterlegen. Beide Möglichkeiten können dann noch mit den Informationen des Flugplans „überschrieben" werden.

Die folgende Tabelle beinhaltet den Ressourcenbedarf an Mitarbeitern mit der Qualifikationsstufe „LM“. Bleibt die Airline-Spalte frei, dann ist dies der Standard-Vorgabewert (Auszug):

\begin{tabular}{|c|c|c|c|c|c|c|}
\hline Flugzeugtyp & Airline & LM & vA & $n A$ & $v D$ & $n D$ \\
\hline A320 & & 1 & 10 & 10 & 40 & 5 \\
\hline A320 & AB & 1 & 15 & & 50 & \\
\hline A320 & XY & 1 & 0 & 10 & 30 & \\
\hline B737 & & 1 & 10 & & 40 & 5 \\
\hline B737 & AB & 1 & 15 & & 50 & \\
\hline
\end{tabular}

Diese Tabelle gibt es für jede Qualifikationsstufe der Mitarbeiter (LM, FA und LD).
LM: Anzahl der benötigten Loadmanager

vA: Vorbereitungszeit vor der Ankunft in Minuten nA: Nachbereitungszeit nach der Ankunft in Minuten vD: Vorbereitungszeit vor dem Abflug in Minuten nD: Nachbereitungszeit nach dem Abflug in Minuten 
Der wöchentliche Flugplan spezifiziert den Planungsgegenstand (Auszug):

Beispiel:

Eine B737 der Airline „XY“ landet am Montag, Mittwoch und Freitag um 10:00 Uhr aus „FRA“ kommend und startet wieder um 12:00 Uhr nach "CNG“

\begin{tabular}{|c|c|c|c|c|c|c|c|c|c|c|c|c|c|c|}
\hline Flugzeugtyp & Airline & $\mathrm{M}$ & D & $\mathrm{M}$ & D & $F$ & S & S & A & A\# & from & $\mathrm{D}$ & D\# & to \\
\hline B737 & $X Y$ & $x$ & & $x$ & & $x$ & & & $10: 0$ & 3222 & FRA & $12: 00$ & 4432 & CNG \\
\hline
\end{tabular}

Es ist anzumerken, dass eine individuelle Zuordnung der Mitarbeiter zu realen Personen nicht Zielsetzung dieser Personaleinsatzoptimierung ist. Es wird „nur“ der kostenminimale Bedarf ermittelt. Somit werden weder eine Mindeststundenanzahl noch ein individualisiertes Stundenkonten für die Mitarbeiter benötigt. Dies ist allerdings mit relativ geringem Aufwand nachträglich zu realisieren. Im Fokus stehen daher die Handhabbarkeit des Verfahrens sowie die Möglichkeit, Trendrechnungen für unterschiedliche Mindestarbeitsstunden je Schicht zu evaluieren.

Die Arbeitszeit der Mitarbeiter kann zu einer beliebigen Viertelstunde beginnen also z. B. um 8:00 Uhr, 8:15 Uhr, 8:30 Uhr, etc.

Die Ausgabe der Optimierungslösung erfolgt in folgender Struktur (Auszug):

\begin{tabular}{|c|c|c|c|}
\hline Wochentag & Qualifikation & Beginn & Dauer \\
\hline Montag & LM & $06: 00$ & 4,5 Stunden \\
\hline Montag & LM & $06: 00$ & 4,5 Stunden \\
\hline Montag & FA & $06: 00$ & 4,5 Stunden \\
\hline Montag & LD & $06: 00$ & 4,5 Stunden \\
\hline Montag & LD & $07: 15$ & 4,0 Stunden \\
\hline
\end{tabular}

\section{» III. MATHEMATISCHE MODELLFORMULIERUNG}

Der Flugplan und Ressourcenbedarf an Mitarbeitern werden zu einer Bedarfstabelle zusammengeführt.

\section{Indexmengen und Indizes}

Q: Menge der Qualitätsniveaus der Mitarbeiter, $\mathrm{q} \in \mathrm{Q}$

T: Menge der Zeitperioden a 15 Minuten einer Woche, $\mathrm{t} \in \mathrm{T}$

$\mathrm{T}_{\mathrm{d}}$ : Menge der Zeitperioden $\mathrm{t}$, die d-1 Zeitperioden vor $t$ liegen

D: Menge der Arbeitszeiten (Dauer in $1 / 4$-Stunden) eines Mitarbeiters, $\mathrm{d} \in \mathrm{D}$
$\mathrm{C}_{\mathrm{q}, \mathrm{t}, \mathrm{d} \text { : }}$

Daten

Bedarf $f_{\mathrm{q}, \mathrm{t}}$ Anzahl an Mitarbeitern mit dem Qualitätsniveau $q$ in der Zeitperiode $\mathrm{t}$

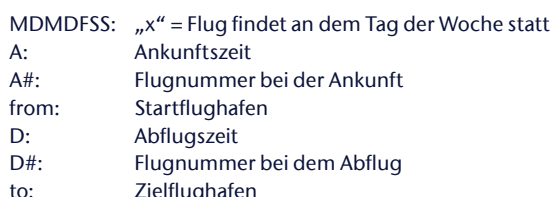

Anmerkung zur Lösungsausgabe:

Es hätte auch eine weitere Spalte mit der Anzahl der benötigten Mitarbeiter eingefügt werden können, der Vorteil dieser Lösung ist es allerdings, dass hierbei gleich in einer weiteren Spalte der Name des Mitarbeiters eingetragen werden kann.

Zielfunktion

$\min \sum_{\mathrm{q} \in \mathrm{Q}} \sum_{\mathrm{t} \in \mathrm{T}} \sum_{\mathrm{d} \in \mathrm{D}} \mathrm{c}_{\mathrm{q}, \mathrm{t}, \mathrm{d}} \mathrm{y}_{\mathrm{q}, \mathrm{t}, \mathrm{d}}$

\section{Restriktionen}

1. Block - Bedarf an Mitarbeitern ist zu mit dem Qualitätsniveau q, der in der $t$ Zeitperiode beginnt und $d 1 / 4$-Stunden arbeitet inklusive der Nacht-, Feiertags- und Wochenendzuschläge.

M: $\quad$ Maximale Anzahl von benötigten Mitarbeitern

\section{Entscheidungsvariablen}

$\mathrm{y}_{\mathrm{q}, \mathrm{t}, \mathrm{d}}$ : Anzahl der Mitarbeiter mit dem Qualitätsniveau q, die zum Zeitpunkt $t$ anfangen und $d$ 1/4-Stunden arbeiten. decken $\sum_{\mathrm{d} \in \mathrm{D}} \sum_{\mathrm{t} \in \mathrm{T}_{\mathrm{d}}} \mathrm{y}_{\mathrm{q}, \mathrm{t}, \mathrm{d}} \geq \operatorname{Bedarf}_{\mathrm{q}, \mathrm{t}}$

für alle $\mathrm{q} \in \mathrm{Q}, \mathrm{t} \in \mathrm{T}$

Nichtnegativitäten

$\mathrm{y}_{\mathrm{q}, \mathrm{t}, \mathrm{d}} \in\{0,1,2, \ldots, \mathrm{M}\}$

für alle $q \in Q, t \in T, d \in D$ 
Der Modellgenerator und die Lösungsaufbereitung wurden in FORTRAN implementiert. Die Verwaltung der Informationen und die Benutzerschnittstelle wurden mit einem handelsüblichen Tabellenkalkulationsprogramm realisiert. Das mathematische Optimierungssystem MOPS (Suhl 1994) in der Version 9.25 diente zur Lösung der Modelle auf einem Intel Core Duo 2,81 GHz, 4GB RAM.

Es sind drei unterschiedliche mathematische Modelle evaluiert worden, die sich bezüglich der Mindestdauer der Arbeitszeit unterscheiden: 3, 31ำ sowie 4 Stunden (h). Die Datenbasis stammt aus dem Ausschnitt eines einwöchigen realen Flugplans mit insgesamt 50 Ankünften und 49 Abflügen.

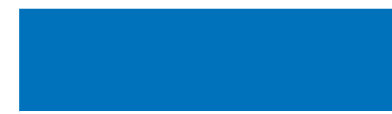

Modelldimensionen: rows $x$ cols $x$ Nonzeros

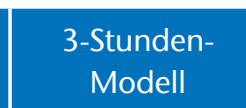

in Tausend:

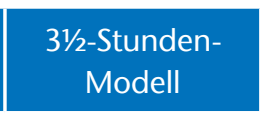

in Tausend:

\section{4-Stunden- Modell}

in Tausend:

\begin{tabular}{|l|c|c|c|}
\hline Lösungszeit in Sekunden: & 0,22 secs & 0,22 secs & 0,11 secs \\
\hline Arbeitszeiten gesamt: & $1.211,5 \mathrm{~h}$ & $1.251,7 \mathrm{~h}$ & $1.303,0 \mathrm{~h}$ \\
\hline davon produktiv: & $704,5 \mathrm{~h}$ & $704,5 \mathrm{~h}$ & $704,5 \mathrm{~h}$ \\
\hline in Prozent: & $58,1 \%$ & $56,3 \%$ & $54,7 \%$ \\
\hline Personalkosten gesamt: & $28.710,36 €$ & $29.703,10 €$ & $30.950,29 €$ \\
\hline relativ zum Günstigsten: & $100 \%$ & $103,5 \%$ & $107,8 \%$ \\
\hline Arbeitseinsatz & & & \\
\hline$\leq 4,5 \mathrm{~h}$ & $92,0 \%$ & $83,8 \%$ & $72,2 \%$ \\
\hline > $4,5 \mathrm{~h}$ und $\leq 6 \mathrm{~h}$ & $8,0 \%$ & $13,9 \%$ & $19,1 \%$ \\
\hline > $\mathrm{h}$ und $\leq 9 \mathrm{~h}$ & - & $2,3 \%$ & $8,7 \%$ \\
\hline > $\mathrm{h}$ & - & - & - \\
\hline Aushilfszeiten & & & $13 \mathrm{~h}$ \\
ein LM als FA: & $10,25 \mathrm{~h}$ & $11,00 \mathrm{~h}$ & - \\
\hline ein LM als LD: & $1,25 \mathrm{~h}$ & $1,75 \mathrm{~h}$ & $1 \mathrm{~h}$ \\
\hline ein FA als LD: & $0,75 \mathrm{~h}$ & $0,25 \mathrm{~h}$ & \\
\hline
\end{tabular}

Anmerkung:

Die Aushilfszeiten geben die Stunden des Arbeitseinsatzes eines Mitarbeiters an, der in einer niedrigeren Qualifikationsstufe tätig ist.

\section{Die detaillierten Ergebnisse je Woche nach Qualifikationsniveau:}

\begin{tabular}{|c|c|c|c|}
\hline Loadmanager & $\begin{array}{l}\text { 3-Stunden- } \\
\text { Modell }\end{array}$ & $\begin{array}{l}\text { 31/2-Stunden- } \\
\text { Modell }\end{array}$ & $\begin{array}{l}\text { 4-Stunden- } \\
\text { Modell }\end{array}$ \\
\hline $\begin{array}{l}\text { Arbeitszeiten - LM ges.: } \\
\text { davon produktiv: } \\
\text { in Prozent: }\end{array}$ & $\begin{array}{c}327,25 \mathrm{~h} \\
185,25 \mathrm{~h} \\
56,6 \%\end{array}$ & $\begin{array}{l}334,25 \mathrm{~h} \\
185,25 \mathrm{~h} \\
55,4 \%\end{array}$ & $\begin{array}{c}345,75 \mathrm{~h} \\
185,25 \mathrm{~h} \\
53,6 \%\end{array}$ \\
\hline $\begin{array}{l}\text { Personalkosten - LM ges.: } \\
\text { relativ zum Günstigsten: }\end{array}$ & $\begin{array}{c}10.057,46 € \\
100 \%\end{array}$ & $\begin{array}{c}10.286,96 € \\
102,3 \%\end{array}$ & $\begin{array}{c}10.659,90 € \\
107,0 \%\end{array}$ \\
\hline Flugabfertiger & $\begin{array}{l}\text { 3-Stunden- } \\
\text { Modell }\end{array}$ & $\begin{array}{l}\text { 31/2-Stunden- } \\
\text { Modell }\end{array}$ & $\begin{array}{l}\text { 4-Stunden- } \\
\text { Modell }\end{array}$ \\
\hline $\begin{array}{l}\text { Arbeitszeiten - FA ges.: } \\
\text { davon produktiv: } \\
\text { in Prozent: }\end{array}$ & $\begin{array}{c}474,75 \mathrm{~h} \\
291,00 \mathrm{~h} \\
61,3 \%\end{array}$ & $\begin{array}{c}493,75 \mathrm{~h} \\
291,00 \mathrm{~h} \\
58,94 \%\end{array}$ & $\begin{array}{l}514,25 \mathrm{~h} \\
291,00 \mathrm{~h} \\
56,6 \%\end{array}$ \\
\hline $\begin{array}{l}\text { Personalkosten - FA ges.: } \\
\text { relativ zum Günstigsten: }\end{array}$ & $\begin{array}{c}11.059,94 € \\
100 \%\end{array}$ & $\begin{array}{c}11.541,19 € \\
104,4 \%\end{array}$ & $\begin{array}{c}12.047,44 € \\
108,9 \%\end{array}$ \\
\hline
\end{tabular}




\begin{tabular}{|l|c|c|c|}
\hline Loader & $\begin{array}{c}\text { 3-Stunden- } \\
\text { Modell }\end{array}$ & $\begin{array}{c}31 / 2-\text { Stunden- } \\
\text { Modell }\end{array}$ & $\begin{array}{c}\text { 4-Stunden- } \\
\text { Modell }\end{array}$ \\
\hline Arbeitszeiten - LD ges.: & $409,50 \mathrm{~h}$ & $423,75 \mathrm{~h}$ & $443,00 \mathrm{~h}$ \\
\hline davon produktiv: & $228,25 \mathrm{~h}$ & $228,25 \mathrm{~h}$ & $228,25 \mathrm{~h}$ \\
\hline in Prozent: & $55,7 \%$ & $53,9 \%$ & $51,5 \%$ \\
\hline Personalkosten - LD ges.: & $7.592,95 €$ & $7.874,95 €$ & $8.242,95 €$ \\
relativ zum Günstigsten: & $100 \%$ & $103,7 \%$ & $108,6 \%$ \\
\hline
\end{tabular}

Es ist auch ein Datendeck mit 1.314 An- und Abflügen berechnet worden. Die Dimensionen des 3-Stunden-Modells betrugen (row $x$ cols $x$ nonzeros in Tausend):

$1,5 \times 52,9 \times 2.049,3$ und wurden in weniger als einer Minute gelöst. Die Arbeitsproduktivität erreichte dabei ca. $90 \%$.

\section{» V. ZUSAMMENFASSUNG UND AUSBLICK}

Aufgrund der hohen Planungskomplexität der realen BVD-Personaleinsatzplanung ist die Verwendung von exakten mathematischen Methoden möglich. Es sind signifikante Unterschiede für die verschiedenen Mindestschichtdauern am Beispiel der Be- und Entladung von Flugzeugen berechnet worden.

In einem weiterentwickelten Modell sollten auch Szenariorechnungen ermöglicht werden, z. B. um erwartete Ereignisse vorauszuplanen. Zu Bedenken ist selbstverständlich auch die Problematik von Verspätungen im Flugbetrieb.

Besonders interessant wären hierbei ex-poste-Analysen, die eine optimale Personaleinsatzplanung nachträglich berechnen - also nicht auf Basis der geplante Abfertigungen sondern der realen Ankunfts- und Abflugdaten.

Ein Soll-Ist-Abgleich könnte in einem wissensbasierten System gespeichert werden und für zukünftige Szenariorechnungen planerische De- und Eskalationsfaktoren bereitstellen. Dies sollte zu einer weiteren Verbesserung des betriebswirtschaftlichen Gesamtergebnisses beitragen.

\author{
LITERATURVERZEICHNIS \\ Suhl U. H. (1994): MOPS - Mathematical OPtimization \\ System. In: European Journal of Operational Research 72, \\ 1994, 312-322. \\ Newsroom ADV deutsche Verkehrsflughäfen (2012a): \\ Mitarbeiter von Europas Flughäfen wehren sich gegen \\ Lohndumping - Flughafenverband ADV weist Brüsseler \\ Liberalisierungspläne entschieden zurück \\ http://www.presseportal.de/pm/44169/2283654/ \\ mitarbeiter-von-europas-flughaefen-wehren-sich- \\ gegen-lohndumping-flughafenverband-adv-weist vom \\ 01.09.2012 um 9:00. \\ Newsroom ADV deutsche Verkehrsflughäfen (2012b): EU- \\ Gesetz zu Bodenverkehrsdiensten ruft ver-di auf den Plan \\ http://www.presseportal.de/pm/44169/2125970/eu- \\ gesetz-zu-bodenverkehrsdiensten-ruft-ver-di-auf-den- \\ plan-betriebsversammlungen-an-allen-grossen vom \\ 01.09.2012 um 8:00. \\ AUTOR \\ Prof. Dr. Ralf Szymanski \\ TH Wildau [FH] \\ ralf.szymanski@th-wildau.de
}

